\title{
Commutativity of Substitution and Variation in the Actions of Quantum Field Theory
}

\author{
Zhong Chao $\mathrm{Wu} *$ \\ Dept. of Physics, Zhejiang University of Technology, \\ Hangzhou, Zhejiang 310032, China
}

\begin{abstract}
There exists a paradox in quantum field theory: substituting a field configuration which solves a subset of the field equations into the action and varying it is not necessarily equivalent to substituting that configuration into the remaining field equations. We take the $S^{4}$ and FreundRubin-like instantons as two examples to clarify the paradox. One must match the specialized configuration field variables with the corresponding boundary conditions by adding appropriate Legendre terms to the action. Some comments are made regarding exceptional degenerate cases.

PACS numbers: 11.10.-z 04.60.-m 04.90.+e 04.50.+h
\end{abstract}

*Electronic address: zcwu2007@yahoo.com 


\section{INTRODUCTION}

It is well known in quantum field theory that all field equations can be derived from an action principle. A field configuration satisfying the field equations is called a classical solution.

By substituting into the action a field configuration which solves a subset of the field equations, the action becomes a functional of the remaining fields. This "reduced action" would describe the partial theory in the classical background of the substituted field configuration. One also expects that varying the reduced action would result in the same field equations for the remaining variables as those derived from the original complete action.

However, the situation is not so simple. A paradox exists in the literature over the fact that substituting a field configuration which solves a subset of the field equations into the action and varying it is not necessarily equivalent to substituting the same field configuration into the remaining field equations [1].

A similar problem had already been encountered earlier in general relativistic cosmology, where imposition of symmetry on the action was found not necessarily to give the correct field equations [2], i.e., imposing symmetry does not necessarily commute with deriving the field equations from an action principle. The reason for the failure is that the symmetry imposition may interfere with the requirement of vanishing variations at the boundary and hence boundary terms cannot be assumed to vanish.

This problem is very disturbing for quantum field theory. Scientists have never been lucky enough to have an ultimate theory with the first try. Instead, they usually consider the known regime as a classical background, which corresponds to the configuration in the substitution. If the above substitution leads to an incorrect reduced action for the remaining variables, then it is simply hopeless to make any progress based on the reduced action of the partial theory. A typical example of this is the consideration of quantum fields in curved spacetime, in which the metric is assumed to be a solution of the Einstein equations. In the mid-1970s, even though little was known about quantum gravity, some very important progress was made in quantum fields in curved spacetime, the discovery of the Hawking radiation in the black hole background being a prime example.

In this paper we use two examples to show that this paradox can be clarified. These two examples are the $S^{4}$ instanton and the Freund-Rubin cosmological models. The paper can 
be thought of as a generalization of a previous paper [3]. We shall discuss the problem of zero value of the cosmological constant in Section II. In Section III the 4-sphere model will be studied. Section IV will be devoted to the Freund-Rubin models. We summarize and conclude this paper in Section V.

\section{THE COSMOLOGICAL CONSTANT PROBLEM}

The most notable example of this confusion had been associated with the cosmological constant problem in quantum cosmology [4]. The issue has been dealt with in the separate publication due to its special importance [3]. However, it is instructive to give a brief review here. To show that the cosmological constant is probably zero, Hawking considered a cosmological model created from an $S^{4}$ seed instanton with the Euclidean action [4]

$$
I=-\int_{M} d x^{4} g^{1 / 2}\left(\frac{1}{16 \pi}\left(R-2 \Lambda_{0}\right)-\frac{1}{8} F^{\mu \nu \rho \sigma} F_{\mu \nu \rho \sigma}\right),
$$

where $R$ is the scalar curvature of the space $M$ of 4-metrics, $\Lambda_{0}$ represents the contributions of "the bare cosmological constant" and the ground states of all matter fields. Here the contribution from a rank 3 antisymmetric tensor gauge field $A_{\nu \rho \sigma}$ is singled out. It arises naturally in $N=8$ supergravity in four dimensions [5]. $F$ is the field strength of $A_{\nu \rho \sigma}$. We use Planck units in which $c=G=k=\hbar=1$.

The field configuration [1][4]

$$
\sqrt{g} F^{\mu \nu \rho \sigma}=(4 !)^{-1 / 2} \kappa \epsilon^{\mu \nu \rho \sigma}
$$

or

$$
F_{\mu \nu \rho \sigma}=(4 !)^{-1 / 2} \sqrt{g} \kappa \epsilon_{\mu \nu \rho \sigma}
$$

with an arbitrary constant $\kappa$ solves the gauge field equation

$$
F_{; \sigma}^{\mu \nu \rho \sigma}=0 .
$$

Substituting this solution (2)-(3) into the action (1), one can see that the $F^{2}$ term behaves like an effective cosmological constant

$$
\Lambda_{e f f}=\pi \kappa^{2},
$$

and the total cosmological constant is $\Lambda_{\text {total }}=\Lambda_{0}+\Lambda_{\text {eff }}$. For $\Lambda_{\text {total }}$, the radius of $S_{4}$ is $\left(3 / \Lambda_{\text {total }}\right)^{1 / 2}$, and the action is $-3 \pi / \Lambda_{\text {total }}$. Here it is assumed that $\Lambda_{\text {total }}$ is positive. The 
action is the negative of entropy of the created de Sitter spacetime. The relative creation probability of the universe is the exponential of the negative of the action [6]. It follows that the most probable configurations will be those with very small values of $\Lambda_{\text {total }}$, and nature will automatically select the right value of $\kappa$ for this. Therefore, Hawking concluded [4]: "the cosmological constant is probably zero."

However, Duff showed that after substituting the configuration into the Einstein equation, which is derived from the complete action (1), the Einstein equation reads

$$
G^{\mu \nu}=\pi \kappa^{2} g^{\mu \nu}-\Lambda_{0} g^{\mu \nu}
$$

Comparing the reduced action and substituted field equation (6), one finds the total cosmological constant appearing in the action is not the same as that appearing in the field equation [1]. What we truly observe is in (6)!

This dilemma can be resolved by choosing the right representation for the wave function of the universe at the equator of the instanton, where the quantum transition from the Euclidean regime $S^{4}$ to the Lorentzian regime $d S^{4}$ occurs. The instanton should not be simply considered as $S^{4}$, it must be considered as a union of a southern hemisphere joined to its time reversal, the northern hemisphere. The action (1) corresponds to the boundary condition that $A_{\nu \rho \sigma}$ is given at the equator between the two hemispheres for the creation probability calculation. However, $A_{\nu \rho \sigma}$ is the wrong representation, which suffers a discontinuity across the equator. Here, we have set $A_{\nu \rho \sigma}$ to be regular in each hemisphere. Therefore one has to use the right representation, its conjugate variable $\sqrt{g} F^{\mu \nu \rho \sigma}$, which is continuous across the equator. The representation transformation is carried out by adding a Legendre term to the action (1)

$$
I_{\text {Legendre }}=-\int_{\Sigma_{S+N}} d S_{\mu} A_{\nu \rho \sigma} F^{\mu \nu \rho \sigma}
$$

where $\Sigma_{S+N}$ denotes the two equator boundaries for both the southern and northern hemispheres.

The total action is

$$
\begin{gathered}
I_{\text {total }}=-\int_{M} d x^{4} g^{1 / 2}\left(\frac{1}{16 \pi}\left(R-2 \Lambda_{0}\right)-\frac{1}{8} F^{\mu \nu \rho \sigma} F_{\mu \nu \rho \sigma}\right)-\int_{\Sigma_{S+N}} d S_{\mu} A_{\nu \rho \sigma} F^{\mu \nu \rho \sigma} \\
=-\int_{M} d x^{4} g^{1 / 2}\left(\frac{1}{16 \pi}\left(R-2 \Lambda_{0}\right)+\frac{1}{8} F^{\mu \nu \rho \sigma} F_{\mu \nu \rho \sigma}\right)
\end{gathered}
$$


where the second equality is obtained by taking divergence of the Legendre term and using the gauge field equation (4).

Substituting (2)-(3) into (8) yields

$$
I_{\text {total }}=-\int_{M} d x^{4}\left(\frac{g^{1 / 2}}{16 \pi}\left(R-2 \Lambda_{0}\right)+\frac{1}{8} g^{1 / 2} \kappa^{2}\right) .
$$

Apparently, varying the action (9) with respect to the gravitational field will result in the same Einstein equation (6). Therefore, Duff's dilemma about the cosmological constant is dispelled and Hawking's argument is completely proven [3].

Two decades after the publication of Hawking's paper, many people believe that the cosmological constant is not zero. Therefore, Duff's paradox has remained long forgotten.

\section{THE 4-SPHERE MODEL}

However, the paradox as a whole has not been resolved. If one is dealing with the simple $S^{4}$ instanton without any boundary, instead of the southern hemisphere joined to the northern hemisphere of $S^{4}$ in the above quantum creation scenario, then it seems that there is no reason to reject the representation $A_{\nu \rho \sigma}$. Even for the representation $\sqrt{g} F^{\mu \nu \rho \sigma}$ it seems that no Legendre term has to be added since there seems to be no boundary in the $S^{4}$ instanton model. Therefore, the dilemma still persists.

Now, let us study this simple $S^{4}$ model with the action (1). As one derives the Einstein and gauge field equations from the action, it is implicitly assumed that the metric $g_{\mu \nu}$ and the gauge potential $A_{\mu \nu \rho}$ are independent variables. In varying the action, one imposes the condition that $A_{\mu \nu \rho}$ is fixed at the boundary.

There is no boundary for the metric of the $S^{4}$ model. People may wonder, why we should bother with the boundary value problem here. The point is that the gauge potential is involved. Since one has to use at least two patches to cover the manifold for the gauge

potential, the Legendre term should arisen from the boundary between the patches under some circumstances. In fact, here we are not particularly interested in varying the action with respect to the gauge field, it has been done and the solution (2) was already found for (4). The key point is that the boundary term for the gauge field is also crucial for our main motivation, the next step: varying the reduced action with respect to the remaining variable, the gravitational field. 
For convenience, the reduced action obtained from substituting (2)-(3) into (1) can be written as follows

$$
I=-\int_{M} d x^{4}\left(\frac{g^{1 / 2}}{16 \pi}\left(R-2 \Lambda_{0}\right)-\frac{1}{8} g^{-1 / 2} \hat{\kappa}^{2}\right),
$$

where $\hat{\kappa} \equiv g^{1 / 2} \kappa$. Now one can vary the reduced action (10) with respect to the gravitational field under the condition that $A_{\mu \nu \rho}$ is fixed. The condition is equivalent to $F_{\mu \nu \rho \sigma}$ being fixed, i.e, $\hat{\kappa}$ is fixed using (3). Here, for simplicity, the minisuperspace ansatz is imposed. This results in the Einstein equation (6), as expected.

One can equally vary the reduced action with respect to the gravitational field under the condition that the conjugate variable $\sqrt{g} F^{\mu \nu \rho \sigma}$ is fixed. It is noted that the variables $\sqrt{g} F^{\mu \nu \rho \sigma}$ and $F_{\mu \nu \rho \sigma}$ are not equivalent in the presence of a gravitational field. Here, we are using $g_{\mu \nu}$ and $\sqrt{g} F^{\mu \nu \rho \sigma}$ as independent variables. For consistency, one has to change the boundary condition correspondingly, that is, add a proper Legendre term to the action.

As emphasized earlier, for the gauge potential $A_{\mu \nu \rho}$, it is impossible to cover the manifold $S^{4}$ by one patch. We can decompose the manifold into two parts: a small ball $B$ and $S^{4}-B$, which we denote as $A$. Following the above procedures, the Legendre term is

$$
I_{\text {Legendre }}=-\int_{\partial A+\partial B} d S_{\mu} A_{\nu \rho \sigma} F^{\mu \nu \rho \sigma} .
$$

One can use the gauge field equation (4) to reduce the Legendre surface term to a divergence term as in (8). Here, we set $A_{\nu \rho \sigma}$ to be regular in $A$ and $B$, respectively. There is an unavoidable discontinuity of the potential across the boundary between them. It turns out that the contributions of the Legendre terms are $-\kappa^{2} V_{B} / 4$ and $-\kappa^{2} V_{A} / 4$, where $V_{B}$ and $V_{A}$ are their volumes. The total contribution of these two Legendre terms for the instanton is $-\kappa^{2} V_{S^{4}} / 4$.

Now, we can let $B$ shrink to a point, then the contributions of $B$ to both the volume and boundary terms of the action tend to zero, and we can discard them. In any case, the total action for $S^{4}$ is (8)-(9). This is the same as that in the model for the cosmological constant problem. The Einstein equation can be derived from the reduced action in the same way.

Indeed, a byproduct of the above argument is that we have proven that it is impossible to regularly express $A_{\mu \nu \rho}$ for the whole $S^{4}$ in one piece or a single gauge. Otherwise, the Legendre term would vanish after shrinking, and this would lead to a contradiction. Therefore, even though $S^{4}$ has no boundary, the inevitable singularity of $A_{\mu \nu \rho}$ leads to the Legendre term. 
In the following, for simplicity, we use gauge freedom to force the singularity to be located at the center of $B$. This is also true for the $S^{n}(n \geq 2)$ model with a rank $(n-1)$ antisymmetric tensor Abelian gauge field.

In summary, what we did is to cover $S^{4}$ by the two patches: $A$ and $B$. For the gauge potential, one can only cover it by at least two arbitrary patches. If we use the representation $A_{\mu \nu \rho}$, we have to tolerate the discontinuities across the boundaries of these patches. If we prefer using the representation $\sqrt{g} F^{\mu \nu \rho \sigma}$ to avoid these discontinuities, then we have to add the Legendre terms, whose total contribution takes the same form as (11), where $\partial A+\partial B$ is replaced by all these boundaries. The total action remains the same as (8). Our choice is just for simplicity. There is no topology change when one lets $B$ shrink to a point. The same arguments apply to the next model. However, for the wave function of the universe only the representation $\sqrt{g} F^{\mu \nu \rho \sigma}$ can be used.

\section{THE FREUND-RUBIN MODELS}

Next turn to a more complicated model, the Freund-Rubin-like instanton [7]. This model has been used to investigate dimensionality of spacetime in quantum cosmology [8]. The spacetime manifold $M$ is described by a product of two spheres $S^{s} \times S^{n-s}$. Apparently, the $S^{4}$ model is a special case with $n=s=4$. The seed for quantum creation of a black hole or a codimension-2 braneworld [9] is an instanton of topology $S^{s} \times S^{2}$, where $S^{2}$ can be a distorted sphere with some conical singularities. It is trivial to generalize our discussion to this case. For simplicity, we shall only consider the Freund-Rubin-like instanton, and the action takes the form

$$
I=-\int_{M} d x^{n} g^{1 / 2}\left(\frac{1}{16 \pi}(R-2 \Lambda)-\frac{1}{2 s} F^{2}\right)-\frac{1}{8 \pi} \int_{\partial M} d x^{n-1} h^{1 / 2} K,
$$

where $R$ is the scalar curvature of $M, K$ is the extrinsic curvature of the boundary $\partial M$ with induced metric $h_{i j}, \Lambda$ is the cosmological constant, and $F$ is the field strength of a rank $-s-1$ antisymmetric Abelian tensor $A_{\alpha_{1} \cdots \alpha_{s-1}}$. We shall use the ansatz that all indices of nonvanishing $F$ components should reside in $S^{s}$. The Euclidean action is obtained via an analytic continuation from the Lorentzian action. There is some overall sign ambiguity in the action. The ambiguity can be eliminated by the following consideration. In order for the primordial fluctuations to take the ground states allowed by the Heisenberg uncertainty 
principle [10], the term associated with the scalar curvature of the external factor spacetime must be negative. But this ambiguity will not affect our discussion.

Similarly, the gauge field equation is

$$
F_{; \alpha_{s}}^{\alpha_{1} \cdots \alpha_{s}}=0 \quad\left(1 \leq \alpha_{i} \leq n\right)
$$

and under the ansatz the solutions must take the form

$$
F^{\alpha_{1} \cdots \alpha_{s}}=\left\{\begin{array}{cc}
(s ! g)^{-1 / 2} \kappa \epsilon^{\alpha_{1} \cdots \alpha_{s}} & \left(1 \leq \alpha_{i} \leq s\right) \\
0 & \text { otherwise }
\end{array}\right.
$$

where $g$ is the metric determinant of the product space, and $\kappa$ is an arbitrary constant. It follows that

$$
F_{\alpha_{1} \cdots \alpha_{s}}=\left\{\begin{array}{cr}
\left(s ! g_{n-s}\right)^{-1 / 2}\left(g_{s}\right)^{1 / 2} \kappa \epsilon_{\alpha_{1} \cdots \alpha_{s}} & \left(1 \leq \alpha_{i} \leq s\right) \\
0 & \text { otherwise }
\end{array}\right.
$$

where $g_{s}$ and $g_{n-s}$ are the metric determinants of the factor spaces.

The Einstein equation is

$$
G^{\mu \nu}=8 \pi \theta^{\mu \nu}-\Lambda g^{\mu \nu}
$$

where the stress tensor is

$$
\theta^{\mu \nu}=F_{\alpha_{1} \cdots \alpha_{s-1}}^{\mu} F^{\alpha_{1} \cdots \alpha_{s-1} \nu}-\frac{1}{2 s} F_{\alpha_{1} \cdots \alpha_{s}} F^{\alpha_{1} \cdots \alpha_{s}} g^{\mu \nu}
$$

From the Einstein equation one can derive the scalar curvatures of the factor spaces

$$
\begin{gathered}
R_{s}=\frac{(n-s-1) 8 \pi \bar{\kappa}^{2}}{n-2}+\frac{2 s \Lambda}{n-2}, \\
R_{n-s}=-\frac{(s-1)(n-s) 8 \pi \bar{\kappa}^{2}}{s(n-2)}+\frac{2(n-s) \Lambda}{n-2},
\end{gathered}
$$

where $\bar{\kappa} \equiv\left(g_{n-s}\right)^{-1 / 2} \kappa$, and it is noted that $\bar{\kappa}$ depends on the metric of the factor space $S^{n-s}$. From these curvatures one can derive the radii of the factor spheres.

Now we substitute the gauge field configuration (14)-(15) into the action (12), then the reduced action is

$$
I=-\frac{1}{16 \pi} \int_{M} d x^{n}\left(\left(g_{s}\right)^{1 / 2}\left(g_{n-s}\right)^{1 / 2}(R-2 \Lambda)-\frac{8 \pi}{s}\left(g_{s}\right)^{-1 / 2}\left(g_{n-s}\right)^{1 / 2} \hat{\kappa}^{2}\right) .
$$

where $\hat{\kappa} \equiv\left(g_{n-s}\right)^{-1 / 2}\left(g_{s}\right)^{1 / 2} \kappa$. 
One can easily derive the Einstein equation from (20) without a boundary term, considering $A_{\alpha_{1} \cdots \alpha_{s-1}}$ or $\hat{\kappa}$ as the given independent variable, as we did in the $S^{4}$ model,

$$
\begin{gathered}
G^{\mu \nu}=-\Lambda g^{\mu \nu}+\frac{4 \pi \bar{\kappa}^{2} g^{\mu \nu}}{s},(1 \leq \mu, \nu \leq s), \\
G^{\mu \nu}=-\Lambda g^{\mu \nu}-\frac{4 \pi \bar{\kappa}^{2} g^{\mu \nu}}{s},(s+1 \leq \mu, \nu \leq n),
\end{gathered}
$$

which is exactly the same as that derived from the complete action (12). Eqs. (18)-(19) are implied by (21)-(22), of course.

One can also derive these from (20) with the Legendre term, considering $\sqrt{g} F^{\alpha_{1} \cdots \alpha_{s}}$ as the independent variable. As usual, the corresponding Legendre term is

$$
I_{\text {Legendre }}=-\int_{\left(\partial \bar{S}^{s}\right) \times S^{n-s}} d S_{\alpha_{1}} A_{\alpha_{2} \cdots \alpha_{s}} F^{\alpha_{1} \cdots \alpha_{s}},
$$

where $\bar{S}^{s}$ denotes $S^{s}$ minus a point where the singularity of $A_{\alpha_{1} \cdots \alpha_{s-1}}$ is located, as in the $S^{4}$ model. The integral is over (the $A$ singularity in $\left.S^{s}\right) \times S^{n-s}$ (in the sense of shrinking $B$ to its center).

Using the gauge field equation (13), the Legendre term can be written

$$
I_{\text {Legendre }}=-\int_{S^{s} \times S^{n-s}} d x^{n} g^{1 / 2} \frac{F^{2}}{s},
$$

where

$$
F^{2}=F_{\alpha_{1} \cdots \alpha_{s}} F^{\alpha_{1} \cdots \alpha_{s}}=\left(g_{n-s}\right)^{-1} \kappa^{2}
$$

The total action is

$$
I_{\text {total }}=-\frac{1}{16 \pi} \int_{M} d x^{n} g^{1 / 2}\left(R-2 \Lambda+\frac{8 \pi}{s} F^{2}\right) .
$$

Using (25), one can recast (26) into the form

$$
I_{\text {total }}=-\frac{1}{16 \pi} \int_{M} d x^{n}\left(\left(g_{s}\right)^{1 / 2}\left(g_{n-s}\right)^{1 / 2}(R-2 \Lambda)+\frac{8 \pi}{s}\left(g_{s}\right)^{1 / 2}\left(g_{n-s}\right)^{-1 / 2} \kappa^{2}\right) .
$$

Varying the reduced total action (27) with respect to the metric for the given independent $\kappa$, one can also derive the same Einstein equation (21)-(22). Nature is always self-consistent!

\section{CONCLUSION}

For a general case, if an original solution is extremal to the original action, then the solution obtained from the reduced action should be extremal too, as long as we supplement 
the action with an appropriate boundary term. However, if the complete solution is nonextremal stationary to the original action, one has to be cautious, the solution that solves the subset of the equations might trace an orbit with constant action value, then the whole orbit may be the solution set for the reduced action and the original solution for the complete action would be one in the solution set after the substitution. We call this case degenerate. Since the Euclidean action of gravitational field is not positive definite, so one has to pay close attention to it. However, this issue does not affect our models due to our choice of variables.

In summary, we have used two models to show that substituting the configuration into the action and then varying it is equivalent to substituting it into the field equation, except for the above degenerate case. The key point is to identify the variables substituted and provide the corresponding boundary conditions, which are implemented by adding to the action the proper Legendre terms needed. In general, the choice of independent variables is made based on convenience. The origin of the inconsistency in the earlier literature is the mismatch of the substituted fields and their boundary conditions. It is believed that as long as we keep this in mind, everything is consistent and the reduced action is viable for the partial theory.

\section{Acknowledgement}

I would like to thank G.W. Gibbons and D. Lohiya for helpful discussions. This work is supported by NSFC No.10703005 and No.10775119.

[1] M. Duff, Phys. Lett. B $\underline{226}, 36-38$ (1989).

[2] R.T. Jantzen, Phys. Rev. D $\underline{34}, 424-433$ (1986).

[3] Z.C. Wu, Phys. Lett. B $\underline{659}$, 891-893 (2008), gr-qc/0709.3314.

[4] S.W. Hawking, Phys. Lett. B134, 403-404 (1984).

[5] E. Cremmer, B. Julia, and J. Scherk, Phys. Lett. B므, 409-412 (1978).

[6] J.B. Hartle, and S.W. Hawking, Phys. Rev. D2ㅇ, 2960-2975 (1983).

[7] G.O. Freund, and M.A. Rubin, Phys. Lett. B $\underline{97}$, 233-235 (1980). 
[8] Z.C. Wu, Gene. Relativ. Grav. 34, 1121-1128 (2002), hep-th/0105021; Z.C. Wu, Phys. Lett. B.585, 6-10 (2004), hep-th/0309178; Z.C. Wu, Gene. Relativ. Grav. 38, 381-386 (2006), gr-qc/0605012.

[9] S.W. Hawking, and S.F. Ross, Phys. Rev. D卢, 5865-5876 (1995); R.B. Mann, and S.F.

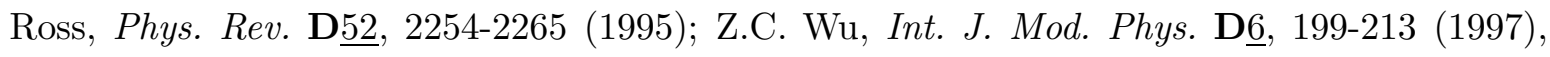
gr-qc/9801020; Z.C. Wu, Phys. Lett. B $\underline{445}$, 274-278 (1999), gr-qc/9810012; Z.C. Wu, Phys. Lett. B $\underline{612}$, 115-121 (2005), hep-th/0405249.

[10] J.J. Halliwell, and S.W. Hawking, Phys. Rev. Dㅆ1, 1777-1791 (1985). 